\title{
Christiane Wiesenfeldt
}

\section{Ritus als Kunst? \\ Das »Magnificat« des 15. Jahrhunderts zwischen Psalmodie und Polyphonie}

Wer sich über die Existenz von musikalischen Gattungen im 15. Jahrhundert informieren will, greift in der Regel zunächst zu Tinctoris' Terminorum musicae diffinitorium von 1472. Die dort versammelten Informationen bestehen zwar zumeist aus heute lapidar anmutenden Anmerkungen in geradezu stenographischer Diktion. Das hat die Musikwissenschaft aber selten davon abgehalten, aus Kategorien, die offenbar der Dokumentation des derzeit aktuellen Begriffsfundus dienen, und rein quantitativ assoziierten Kategorien wie »magnus«, »mediocris « und »parvus«, hierarchische Klassifikationen abzuleiten: im Sinne von »erhabener« Größe einer Messe (»missa«), mittlerer, wenn nicht >mittelmäßiger Bedeutung der Motette (»motetus«) und kleiner, eben >mäßiger $<$ Bedeutung des Gesangs (»cantilena«). In diesem häufig missverstandenen Gattungspanorama - so stellt man nach kurzem Blättern fest - ist für das Magnificat jedenfalls kein Platz, es kommt bei Tinctoris schlicht nicht vor. ${ }^{1}$ Allenfalls der Eintrag für Hymnus, das klassische Loblied ohne spezifische Orientierung, ließe sich verwenden. Er lautet bei Tinctoris - in beinahe boshafter Trivialität, so möchte man sagen -: »Hymnus est laus Dei cum cantico«, gefolgt von, noch banaler: »Hymnista est ille qui hymnos canit «. ${ }^{2}$ Die fehlende formale Klassifikation des Hymnus dürfte allerdings kaum auf seine untergeordnete Bedeutung im zeitgenössischen Gattungsspektrum als vielmehr darauf hindeuten, dass die funktionale Determinante des »laus Dei«, des Gotteslobs, hier sein maßgebendes Definitionskriterium darstellt, ein Kriterium zumal, das keiner flankierenden, wo-

1 Terminorum musicae diffinitorium (=Scriptorum de musica medii aevi nova series a Gerbertina altera, Bd.4), hrsg. von Edmond de Coussemaker, Paris, 1864-76, Reprint Hildesheim 1963, S. 177-191. Er erwähnt das Magnificat hingegen in seinem De inventione et usu musicae (ca. 1481), wo es als »canticum hoc egregium« betitelt wird, das man zudem »amoenissime cantavit «, vgl. Karl Weinmann, Johannes Tinctoris (1445-1511) und sein unbekannter Traktat »De inventione et usu musicae". Eine historisch-kritische Untersuchung, Regensburg 1917, S. 27-46: S. 32.

2 Terminorum musicae diffinitorium (wie Anm. 1), S. 184. 
möglich gar ästhetisch misszudeutenden Zusatzkategorie bedarf. Vielmehr ließe sich umgekehrt festhalten, dass die Sammelbegriffe Lied, Motette und Messe, denen Tinctoris keine funktionalen Zuweisungen zudachte, sich offenbar weniger durch ihren funktionalen Kontext als zunächst vielmehr durch ihre formalen Kriterien gliedern lassen. Um dies - und um nicht mehr oder weniger! - ist es diesem Katalog an Definitionen im Diffinitorium zu tun.

Will man sich also einen Eindruck von der Entfaltung, Stellung und dem Wandel des Magnificat im 15. Jahrhundert machen, so bieten sich vielmehr die zahlreichen zeitgenössischen Lehrbücher zum Verfassen und Intonieren eines solchen Werkes an. Hier entfaltet sich schon beim ersten Blick eine geradezu verblüffende Vielfalt an ritueller Kombinatorik, Melodievarianz oder auch regional tradierter Strukturalisierung. Erstaunlicherweise ist dieser kontextualisierende Fundus an Quellen und seine Bedeutung für die Gestaltung und Verbreitung des Magnificat bislang nicht hinreichend ausgewertet worden - die verdienstvollen Studien zum Magnificat im 15. und 16. Jahrhundert von Josef Meinholz, ${ }^{3}$ Karl-Heinz Illing ${ }^{4}$ und insbesondere Winfried Kirsch ${ }^{5}$ dienen in erster Linie der Lokalisierung und Sicherung der mehrstimmigen Überlieferung und/oder ihrer formalen Varianten, nicht aber der frühen (auch: monophonen) Überlieferung in europäischen Repertoires seit dem 10. Jahrhundert, der liturgischen Verortung oder didaktischen Aufbereitung in Choraltraktaten. Die enorme Materialbasis - eine einfache Begriffssuche im Thesaurus Musicarum Latinarum ${ }^{6}$ etwa ergab knapp 300 Treffer bis 1500 - lässt sich in diesem Rahmen freilich nicht einmal andeuten. Hinsichtlich des hier zu diskutierenden Akzents der Normierung und Pluralisierung motettischer Gattungen und Sonderformen im 15. Jahrhundert sind aus den gesichteten Traktaten zumindest zwei wichtige Spannungsfelder des Magnificat festzuhalten, die in grundlegende Fragestellungen zu lenken sind:

1. Die musikalischen Prämissen des Magnificat gelten gemeinhin als vergleichsweise statisch. Sein strophischer Textkorpus ist schließlich - wie in der Messe - stets identisch, und sein Rezitationston scheint den melodischen Ereignisraum, ob ein-, ob mehrstimmig, drastisch einzuengen. Die Traktate geben hingegen nicht nur zahlreiche unterschiedliche Varianten für jene Abschnitte

3 Josef Meinholz, Untersuchungen zur Magnificat-Komposition des 15. Jahrhunderts, Diss. Köln 1956.

4 Karl-Heinz Illing, Zur Technik der Magnificat-Komposition des 16. Jahrhunderts, Wolfenbüttel 1936.

5 Winfried Kirsch, Die Quellen der mehrstimmigen Magnificat-und Te Deum-Vertonungen bis zur Mitte des 16. Jahrhunderts, Tutzing 1966.

6 http://www.chmtl.indiana.edu/tml/start.html (15.8.2011). 
der Melodie, die als initium, meditatio und terminatio jene plan auf dem Rezitationston lagernden tuba-Abschnitte rahmen bzw. unterbrechen (vgl. dazu weiter unten Abbildung 2). Diese variablen Abschnitte sollen zudem bei einer Komposition im anderen Psalmton grundlegend variiert werden - nicht jeder Psalmton bedingt also dieselbe melodische Kontur. Darüber hinaus werden noch im 16. Jahrhundert lediglich die Initien in den Traktaten präsentiert. Wie der Rest der Strophen zu gestalten ist, die zumal sämtlich andere Silbenzahlen aufweisen (vgl. weiter unten Abbildung 1), bleibt dem Ausführenden überlassen. Andere Traktate bieten Varianten der terminatio an; ${ }^{7}$ auch hier scheinen regionale Typologien eine - womöglich niemals tatsächlich existierende - paneuropäische Tradition zu überwölben. In wiederum anderen Traktaten lassen sich Varianten ausmachen, sobald die das Magnificat einrahmenden Antiphonen den Modus und damit das Anfangsintervall ändern, woran das Magnificat anzuschließen hat. Und schließlich ist in der mehrstimmigen Vertonung stets dem Komponisten überlassen, welche der Zeilen er im alternatim-Verfahren polyphon und welche er unison (choraliter) umzusetzen gedenkt, und ob er Strophen anders textiert wiederholen lässt oder ob in einer Strophe die Psalmtonrezitation oder doch die melismatisch agilen Passagen initium, meditatio und terminatio dominieren sollen, ganz zu schweigen von der polyphonen Ausgestaltung der freien Rahmenstimmen. Zunächst lässt sich also festhalten: Weder in seiner Aufbereitung in den Traktaten noch in seiner mehrstimmig überlieferten Form stellt sich das Magnificat des 15. Jahrhunderts als strukturell und formal statisch dar. Dass die Scharniere zu den es umgebenden Teilen der Vesper modal und intervallisch abgestimmt - und somit zugleich von ihnen definiert - werden, verhindert keineswegs, dass der Raum im Magnificat selbst als exponierter Ort der variatio beschrieben werden kann, als ein sich auf mehreren Ebenen zugleich realisierendes Erweiterungsstreben in Modus, Form, Melodik, Besetzung, Satzart und Ausdehnung.

2. Der stets identische funktionale Ereignisort des Magnificat ist die Vesper im täglichen monastischen Offizium, wo es den finalen Höhepunkt markiert. Allein die tägliche Ausführung lässt es kaum geeignet erscheinen, so könnte man meinen, für das Magnificat Kunstwerk-relevante Ansprüche zu formulieren, gelten doch der Musikgeschichtsschreibung noch immer jene Gattungen als besonders kunsthaft, denen es gelungen war, sich von liturgischen Ansprüchen - und

7 Vgl. etwa - als ein Beispiel unter vielen - einen polnischen Musiktraktat des 15. Jahrhunderts: Johannes de Szydlow, Musica magistri Szydlovite (Warschau, nach 1475), http://www.chmtl. indiana.edu/tml/15th/SZYDMUS_TEXT.html (15.8.2011). 
sei es nur partiell durch weltliche Textimplementierung oder das Überblenden von Aussagekraft - zu lösen. In diesem - nebenbei gesagt: dringend revisionsbedürftigen - Definitionsraum dürfte dem Magnificat zweifelsohne eine besonders dienende Funktion zukommen: In täglicher Redundanz an denselben rituellen Ort gebannt, konnte sich wohl kaum ästhetische Relevanz entwickeln. Dass dieses Bild schief und die Reihe der die Vesper begründenden Cantica keineswegs als gebetsmühlenartiges Einerlei im rituellen Pflichtprogramm zu verstehen ist, lässt sich ebenfalls aus gleich mehreren musikalischen Traktaten ablesen. Dort wird stets darauf verwiesen, dass das Magnificat unter den Psalmen eine herausragende Stellung einnimmt, allein schon wegen seiner Ausdehnung: »Maiores vero psalmi sunt $>$ Benedictus Israhel $<,>$ Magnificat anima mea Dominum $<,>$ Nunc dimittis servum «", heißt es etwa in einem anonymen Tractatus de musica plana et mensurabili aus dem 15. Jahrhundert, ${ }^{8}$ und Tinctoris gibt in seinem De inventione um 1481 zu Protokoll: "canticum hoc egregium Magnificat: ab ea (ut prediximus) in novitate spiritus compositum: amoenissime cantavit «. ${ }^{9}$ Vokabeln wie »bemerkenswert", »geistvoll«, »lieblicher Gesang «, etc. verweisen vielmehr auf die hymnische Konnotation dieser bedeutenden marienliturgischen Gattung an sich, auf ihren unbestrittenen devotionalen Rang, der sich nicht trotz, sondern gerade wegen, also eben in ihrer funktionalen Gebundenheit realisierte.

Nimmt man die Fäden dieser beiden hier nur skizzierten Spannungsfelder zusammen, so entsteht vor dem musikhistorischen Horizont eines sich zunehmend pluralisierenden Gattungsspektrums ein merkwürdiges Bild: Eine der funktionalen marianischen Kerngattungen des 15. Jahrhunderts, deren Beitragsdichte im 16. Jahrhundert schließlich massiv zunimmt, arbeitet sich an strukturellen Herausforderungen wie enger Vorlagenbindung, formaler Parzellisierung und extremer Modusfixierung ab. Hierin erzeugt sie allerdings einen in kaum einer anderen Gattung zu beobachtenden Formenreichtum stilistischer und kombinatorischer Verfahren hin zu geradezu hochartifiziellen Kraftakten kompositorischer Gestaltung, die in bloßer Gebrauchsmusik wohl kaum erforderlich gewesen wären. Um sich dieser komplexen Ausgangssituation anzunähern, werden im Folgenden zunächst die religiöse Textbasis, sodann die musikalischen Trends bis 1500 umschrieben, um schließlich nach den Resultaten für die rituelle Funktion einerseits und die musikhistorische Deutung und Verortung der Gattung andererseits fragen zu können.

8 Anonymus XI, Tractatus de musica plana et mensurabili, http://www.chmtl.indiana.edu/tml/ 15th/ANOIITDM_TEXT.html (15.8.2011).

9 De inventione (wie Anm. 1), S. 32. 
Der Magnificat-Text gehört zu den meistdiskutierten des hymnischen Fundus. Das betrifft einerseits die grundlegende Frage, ob Maria tatsächlich die monologisierende Hauptfigur ist oder nicht: Die in altlateinischen Handschriften überlieferte Lesart, wonach der Magnificat-Text nicht von Maria, sondern Elisabeth gesprochen wird, ist immerhin mittlerweile als sekundär erkannt; andere Quellen erörtern zumindest noch Elisabeths mögliche Autorschaft des Textes. ${ }^{10}$ Andererseits sind die Textauslegungen unterschiedlich erfolgt, je nach Gliederung der zwölf Zeilen und je nach Interpretation der marianischen Funktion in der Heilsgeschichte, die seit den Regulae des Heiligen Benediktus aus dem 5. Jahrhundert freilich von Ort zu Ort und von Zeit zu Zeit differierte. Die Handlung des literarisch anspruchsvollen Textes wurzelt in der lukanischen Szene der Begegnung Marias mit Elisabeth (Lukas 1,46-55, vgl. die Aufstellung des Textes in Tabelle 1).

Maria betet ob ihrer Erwählung und erkennt zudem erstmals ihre neue Rolle als institutionalisierte Mittlerin, als Gegenstand der Anbetung: »Beatam me dicent omnes generationes « (Vers 48). Ihre Funktion als Betende - in der mariologischen Fachliteratur gelegentlich hierarchisiert als »Vorbetende «ll - identifiziert sie zugleich mit den Gläubigen der Gemeinde Israel, als deren Urmutter sie gilt. Die zehn Verse werden abschließend durch ein Gloria Patri doxologisch ergänzt und abgeschlossen. Der Text weist - im Gegensatz zu den meisten anderen biblisch inspirierten Hymnica - eine episodische, ja dramaturgische Diktion auf: Er wandelt sich von einem autobiographischen Passus in den ersten vier Versen - »meine Seele", "preisen mich selig «, hat Großes an mir getan" - hin zu einem eigenen, heilsgeschichtlichen Lobpreis von Gottes Macht (Vers 50-53) und sodann seiner Barmherzigkeit (Vers 54f.). ${ }^{12}$ Daran, dass gläubige Bitten oder Ähnliches gänzlich fehlen, ist der rein hymnische, lobpreisende Charakter deutlich ablesbar. Maria ist allerdings zugleich exemplarische Sprecherin des Textes, sie wird - erst recht später in der protestantischen Auslegung des Magnificat-als Vertreterin der Gläubigen angesehen, umso leichter deswegen, da sie selbst als

10 Vgl. aus kirchen- und frömmigkeitsgeschichtlicher Perspektive zum Magnificat unter anderem: Orby Shipley, Carmina Mariana. Second series, London 1902, S.260-263; John Julian, A dictionary of hymnology, setting forth the origin and history of christian hymns of all ages and nations, London ${ }^{2}$ 1907, S.711 (Magnificat); Carl Marbach, Carmina Scripturarum, Straßburg 1907, S.430-433; Dimonic Johner, A New School of Gregorian Chant, Regensburg 1914, S. 60-69; John Singenberger, Guide to Catholic Church Music, St. Francis 1905, S. 148-150.

11 Franz Courth, »Marianische Gebetsformen «, in: Handbuch der Marienkunde, hrsg. von Heinrich Petri und Wolfgang Beinert, Regensburg 1984, S. 336-403: S. 379.

12 Vgl. Art. »Magnifikat", in: Marienlexikon, hrsg. von Remigius Bäumer und Leo Scheffczyk, St. Ottilien 1994, Bd.4, S.235-241. 


\begin{tabular}{|c|c|c|c|}
\hline & Lukas 1, & Text-Struktur & Latein (Vulgata) \\
\hline 1 & $46 b$ & \multirow{4}{*}{$\begin{array}{l}\text { [Aufgesang -] } \\
\text { Lobpreis Gottes für } \\
\text { selbst empfangenes } \\
\text { Heil }\end{array}$} & $\begin{array}{l}\text { Magnificat anima mea Dominum, } \\
\text { et exsultavit spiritus meus in Deo salutari meo. }\end{array}$ \\
\hline 2 & 47 & & Quia respexit humilitatem ancillae suae. \\
\hline 3 & 48 & & Ecce enim ex hoc beatam me dicent omnes generationes. \\
\hline 4 & 49 & & $\begin{array}{l}\text { Quia fecit mihi magna, qui potens est, } \\
\text { et sanctum nomen eius. }\end{array}$ \\
\hline 5 & 50 & \multirow{4}{*}{ Gottes Macht } & $\begin{array}{l}\text { Et misericordia eius a progenie in progenies } \\
\text { timentibus eum. }\end{array}$ \\
\hline 6 & 51 & & $\begin{array}{l}\text { Fecit potentiam in brachio suo, } \\
\text { dispersit superbos mente cordis sui. }\end{array}$ \\
\hline 7 & 52 & & $\begin{array}{l}\text { Deposuit potentes de sede } \\
\text { et exaltavit humiles. }\end{array}$ \\
\hline 8 & 53 & & $\begin{array}{l}\text { Esurientes implevit bonis } \\
\text { et divites dimisit inanes. }\end{array}$ \\
\hline 9 & 54 & \multirow{2}{*}{$\begin{array}{l}\text { Gottes } \\
\text { Barmherzigkeit } \\
\text { [- Abgesang] }\end{array}$} & $\begin{array}{l}\text { Suscepit Israel puerum suum, } \\
\text { recordatus misericordiae suae. }\end{array}$ \\
\hline 10 & 55 & & $\begin{array}{l}\text { Sicut locutus est ad patres nostros, } \\
\text { Abraham et semini eius in saecula. }\end{array}$ \\
\hline $\begin{array}{l}11 \\
12\end{array}$ & & $\begin{array}{l}\text { stets hinzugefügtes } \\
\text { »Gloria Patri« }\end{array}$ & $\begin{array}{l}\text { Gloria Patri et Filio et Spiritui Sancto, } \\
\text { sicut erat in principio et nunc et semper } \\
\text { et in saecula saeculorum. Amen. }\end{array}$ \\
\hline
\end{tabular}

\section{Tabelle 1: Der Text des Magnificat}

ein, wenn auch ausgewählter Mensch gilt. Der Lobpreis, den Maria hier stellvertretend für die Gläubigen betet, weitet sich also von einem Monolog zu einem Gemeinschaftsgebet, vom individuellen zum allgemeinen und sodann, im Gloria Patri, zum allumfassenden, krönenden Vaterunser »in Ewigkeit«. Der dem ganzen innewohnende, emphatische Duktus hat aus ganz verschiedenen Disziplinen über die Jahrhunderte hinweg Deutung erfahren, von der Verwendung des Magnificat als Schlussgebet in exorzistischen Kulten bis hin zur aktuellen feministischen Deutung Dorothee Sölles, die darin bereits das Postulat ausgesprochen sieht, mit der »Herrschaft der Männlichen über die Weibchen« werde es 


\begin{tabular}{|l|l|} 
Deutsch & \\
\hline Meine Seele preist die Größe des Herrn, \\
und mein Geist jubelt über Gott, meinen Retter.
\end{tabular}

alsbald »ein Ende nehmen ${ }^{13}$ Ganz gleich aber, welche Interpretation man dem Text angedeihen lässt: Unstrittig ist seine dramaturgische Anlage, die ihn per se - so möchte man meinen - für eine musikalische Umsetzung prädestiniert.

Ein Blick auf die mehrstimmigen Magnificat-Vertonungen im 15. Jahrhundert zeitigt sodann erwartungsgemäß ebenso vielfältige Ergebnisse, wie die Choraltraktat-Situation bereits ahnen ließ. Konstante im Repertoire bleibt aller-

13 Dorothee Sölle, "Magnifikat", in: Warten, dass er kommt. Advent und Weibnachten. Biblische Texte verfremdet, Bd.2, hrsg. von Sigrid und Horst Klaus Berg, München 1986, S. $31 \mathrm{f}$. 
dings zwangsläufig der (adaptierte oder selbst kreierte) Psalmton-cantus firmus, der eine charakteristische, wenn auch nicht sklavisch beibehaltene Formung pro Strophe erfährt (vgl. Abbildung l).

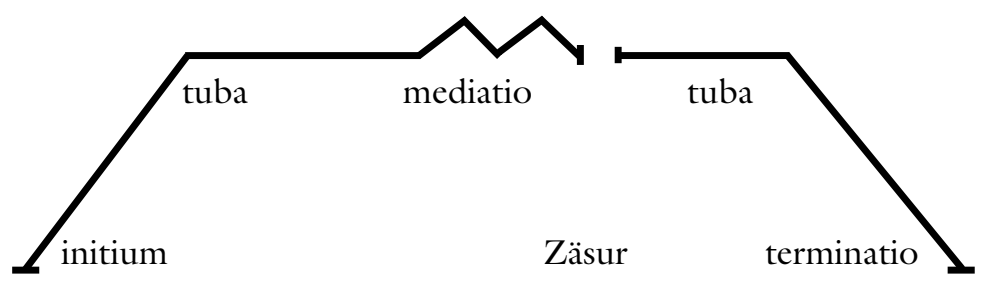

Abbildung 1: Grundstruktur des psalmodischen cantus firmus ${ }^{14}$

Gemäß seiner Anlage ist jeder Psalmvers in zwei Teile gegliedert: initium + tuba mediatio // tuba + terminatio bzw. finalis. Das initium wird nur beim ersten Vers gesungen, die folgenden beginnen mit der tuba. Dabei wird der Rezitationston jeweils entsprechend der Anzahl der nicht von den anderen Melodieteilen abgedeckten Silben des Psalms wiederholt. Die finalis leitet vom Rezitationston zum Anfangston der anschließend gesungenen Antiphon über (zur tonalen Disposition vgl. Tabelle 2 ).

\begin{tabular}{l|l|c|c} 
Psalmton & Kirchenton & tuba & finalis \\
\hline I. & dorisch & $a$ & $d$ \\
\hline II. & hypodorisch & $f$ & $d$ \\
\hline III. & phrygisch & $b / c$ & $e$ \\
\hline IV. & hypophrygisch & $a$ & $e$ \\
\hline V. & lydisch & $c$ & $f$ \\
\hline VI. & hypolydisch & $a$ & $f$ \\
\hline VII. & mixolydisch & $d$ & $g$ \\
\hline VIII. & hypomixolydisch & $c$ & $g$
\end{tabular}

Tabelle 2: Tonale Verortung der Psalmodie

14 Entnommen aus J. Meinholz, Untersuchungen (wie Anm. 3), S. I. 
Die Komponisten des 15. Jahrhunderts gehen mit dieser »Vorgabe", die - wie eingangs erwähnt - allenfalls nur latenten Einfluss auf Melodiegestalt, Länge, Stilmittel und Form des Ganzen hat, erwartungsgemäß unterschiedlich um. Gemeinsam ist ihnen in der Regel nur die Diskantlage des cantus firmus, die sich bisweilen noch im 16. Jahrhundert findet. Ihre Selbstverständlichkeit in der polyphonen Umsetzung korreliert wohl nicht zufällig mit der quasi monodischen, von einer zumal hohen Stimme vorgetragenen Hymnusfaktur. Marias Text wird stimmlich grundiert und nicht umrahmt, und das dafür maßgeblich geeignete Satzmodell ist der insbesondere von Dufay kultivierte Fauxbourdon-Satz. Im Vorfeld dieser Tendenzen zur latenten Profilbildung im Magnificat begegnen freilich zahlreiche Werke - etwa in den vierstimmigen Magnificat von Petrus Johannes de Quatris -, die ihre Verwandtschaft zu genuin motettischen Prinzipien nicht verleugnen können: Eingelagert in den Tenor, läuft der cantus firmus nur in den Rahmenteilen eines Verses nachvollziehbar ab; seine Mitte ist hingegen gekennzeichnet von rhythmisch-melodischer Sektionierung von cantus-firmusAbschnitten, die sowohl homöorhythmische, homorhythmische, isorhythmische als auch isomelodische Kennzeichen aufweisen. Inwieweit diese Beispiele repräsentativ für das Magnificat um 1400 sind, kann aus der schmalen Quellenbasis freilich nicht gefolgert werden, ohne konstruiert zu wirken. Vielmehr lässt sich umgekehrt festhalten, dass motettische Struktur-Prinzipien der Repetition und Binnengliederung sogar in die psalmodische Präsentation im Magnificat Eingang fanden: Das repetierende Moment der Psalmodie wurde hier quasi kompositorisch substantiviert.

Dufays Fauxbourdon-Gestaltung des Magnificat, die für mehrere Dekaden vorbildhaft werden sollte, weist kaum Ansätze zur Parzellierung des cantus firmus auf, der in seiner Zwei- bzw. Vierteiligkeit weitgehend unangetastet bleibt und sich allenfalls an den Zäsuren imitatorisch gelockert gibt. Erst die Generation nach Dufay widmet die vier Hauptteile des cantus firmus immer öfter zu vier Binnenimitations-Abschnitten pro Vers um, die zur Durcharbeitung des Materials neigen und entsprechend nun auch Textpassagen, die nicht auf das jeweilige initium fallen, akzentuieren können. Wortgruppen-Imitationen, Doppelchörigkeit und tonale Verlagerungen sind die angewandten Mittel, die in den Werken Ockeghems und Busnois' erstmals ein mehrfaches Aufbrechen tonaler und formaler Achsen andeuten und schließlich durchsetzen. Die psalmodische Fixierung insbesondere der tuba-Abschnitte wird, wenn nicht aufgehoben, so doch gelockert, und die durch den Textverlauf gesteuerte formale Anlage an beliebigen Punkten geweitet und interpretiert. Hier, etwa um die Mitte des 15. Jahrhunderts, treten im Magnificat immer mehr Individualismen zutage, die 
sich nicht allein aus einer kompositionsgeschichtlichen Weitung der Parameter erklären lassen, erst recht, wenn man die frühen, rhythmisch strukturalisierten Entwürfe um 1400 mitbedenkt, die ja wohl kaum als rudimentäre Vorstadien bezeichnet werden können. Wenn hier mithilfe der zunehmend perfektionierten imitatio-Technik das polyphone Gewebe beginnt, den psalmodischen, homophonen, satztechnisch daher vertikalen Rahmen zu dehnen und in eine horizontal geschichtete Formation umzulenken, dann geschieht das nicht von selbst aus dem Material heraus. Auch wäre es geradezu ahistorisch und der liturgischen Verortung der Gattung unangemessen, von einer absichtsvollen Lösung aus rituellen Verpflichtungen zu sprechen, die der Komponist als Künstler im emphatischen Sinne nun anstrebe. Die Funktion ist die Legitimation dieser, wie jeder anderen geistlichen Gattung, und nicht ihr Antagonist. Was hier mit den neuen Satztechniken im Magnificat sukzessive entsteht, ja sie womöglich erst inspirierte (worüber nur spekuliert werden kann), ist eine latente innere Umwertung des Verhältnisses von Text und Musik, nicht zuletzt von Ritus und Kunst. Waren die heute bekannten frühen Entwürfe des Magnificat um 1400 zwar durch kunstvolle Techniken aufgefallen, in denen anspruchsvolle musikalische Proportionen gestiftet wurden, denen der Text zu folgen hatte, und waren Dufays Entwürfe von dem Bemühen um eine volle klangliche Stütze des Oberstimmen-gelagerten, weitgehend unangetasteten cantus firmus getragen, so drängte ab der Jahrhundertmitte zunehmend die Interaktion von Text und Musik, und damit weitläufig von Ritus und Kunst in den Vordergrund. Die Narrativität der biblischen Szene wird quasi musikalisch erobert. Besonders wirkmächtige Zeilen - wie etwa »Esurientes implevit bonis « - werden in zahlreichen Magnificat semantisch pointiert umgesetzt, tonale Erweiterungen des Psalmton-Raumes bis hin zu seiner partiellen Suspendierung verraten - erst recht in Steigerungsabschnitten - dramaturgische Absichten, und die geradezu ausufernde formale Variantenbildung durch Parzellierung, Kombinatorik und Wiederholungen von Abschnitten deuten auf individuelle Akzentuierungen des Textes hin.

Diese beinahe schon improvisiert klingende Struktur des Magnificat, die sich im 15. Jahrhunderts auszuprägen begann und sich im 16. Jahrhundert quasi pluralisierte, mündete hingegen nicht oder nur äußerst selten in zwei- bis dreiteilige Großformen, ${ }^{15}$ die man getrost als Motette bezeichnen könnte. Das liegt weniger an der strukturellen Qualifikation der Gattung als an der ihr invarianten funktionalen Bindung an den strophisch geformten Text, der wegen der rituel-

15 Beispiele aus dem 16. Jahrhundert dafür wären die Magnificat von Gasparo Alberti oder Valentin Rab. 
len Konstante keine Kürzungen oder Interpolationen erlaubte. Ganz gleich, wie akzentuiert mancher Vers gestaltet und welches interpretatorische Übergewicht ihm damit gegeben sein mochte, der Verlauf der zwölf Verse blieb zwingend, ob alternierend oder auskomponiert.

Dass die skizzierten Entwicklungen in der Magnificat-Interpretation im 15. Jahrhundert Konsequenzen für ihre rituelle Funktion hatten, der sie entstammten und stets zugehörten, ist sicher unbestritten. Diese Konsequenzen sind hingegen - wie bereits mehrfach angedeutet - kaum funktions-störend, sondern stets -befördernd gewesen. Das betrifft im Wesentlichen drei Aspekte:

Durch den Pluralismus in der Magnificat-Auslegung im 15. Jahrhundert wurde eine Variabilität im Tagesprotokoll geschaffen, die zwar stets funktionale Garantie bot, zugleich aber eine ästhetisch ansprechende Abwechslung generierte, eine Abwechslung, die einem literarischen Textgehalt angemessen war, der als »marianisches Hohelied « galt und mit Konnotationen wie »bemerkenswert «, »geistvoll«, »lieblich « geradezu nach multipler künstlerischer Huldigung verlangte.

Diese »Individualisierung des Ritus « machte schließlich eine künstlerische Profilbildung möglich, was spätestens um 1500 als ein wesentlicher Antrieb eines Komponisten gelten durfte. Indem das Magnificat neben dem rein kirchlichen Ritus auch zunehmend Medium privater Andachtsräume wurde, konnten diese künstlerischen Profile auch vervielfältigt und exportiert werden, was der Ruhmsicherung diente. Und schließlich, indem Marias Gebet, das als Vorgebet zugleich allen in den Mund gelegt ist, allegorische Qualität qua musikalischer Interpretation erhält, steigert dies die symbolische Vermittelbarkeit der Aussage an den Adressaten respektive die Gemeinde. Marias Vermittlerkompetenz, ihre Funktion als »mediatrix " zwischen Gott und den Menschen, wird dadurch akzentuiert, und mit ihr zugleich - und das ist entscheidend - der ekklesiale Aspekt: Maria als »mater ecclesia ", als Identitätsfigur der Institution Kirche selbst, erhält damit umso mehr Profil und Wirkungsmacht.

Für die Deutung des Magnificat aus musikologischer Perspektive haben diese enge Verwobenheit von Funktion und Struktur, von Text und Musik, von Ritus und Kunst ebenfalls nicht unerhebliche Konsequenzen. Die isolierte Betrachtung nur der kompositorischen Entwicklung vernachlässigt nicht nur den engen $\mathrm{Zu}$ sammenhang der sich im Spannungsfeld von Psalmodie und Polyphonie ereignenden Interpretation. Darüber hinaus tendiert sie beinahe zwangsläufig zu einer teleologischen Deutung, die Kontinuitäten und Normen konstruiert, um 
der Pluralität von Erscheinungsformen, die nicht zuletzt in den Choraltraktaten dokumentiert ist, mit komplexitätsreduzierender Systematik zu begegnen. Dies erscheint gerade im Magnificat, das sich gegenüber Rubrizierungen allzu sperrig erweist, denkbar ungeeignet: Polare Differenzierungen wie Norm und Abweichung, Zentrum und Peripherie, Universalismus und Regionalismus greifen hier schlicht zu kurz.

Das lässt sich an einer Kategorie am besten demonstrieren, die im Magnificat quasi ad absurdum geführt wird, betrachtet man sie allein aus musikalischer Perspektive: der Zyklus. Vielmehr wäre, wenn überhaupt, von gleich mehreren zyklischen Ebenen zu sprechen: dem >kleinen`Zyklus der Versvertonung mit seiner zyklischen, kreisförmig zur finalis des Psalmtons zurückkehrenden Form, dem >mittleren< Zyklus eines Magnificat mit den zwölf Versen in alternierender oder gar abwechselnd wiederkehrender Strophenform und schließlich dem >großen $<$ Zyklus - und in dieser Definition ist er für das Magnificat am gebräuchlichsten -, der eine zyklische Werkgruppe des Magnificat in allen acht Psalmtönen in systematischer Stufenfolge meint. Auf allen drei Ebenen mögen fraglos Aspekte des Zyklischen - sei es die Finalbezogenheit, seien es motivische, formale oder besetzungstechnische Proportionen, seien es tonale Verlaufsskizzen - eine Rolle spielen. Von einem großformal ambitionierten, >zyklischen< Magnificat zu sprechen, ginge jedoch an der Sachlage vorbei. Nicht zuletzt deshalb existieren beim Magnificat jene schon bei der >zyklischen< Messe verwundert beäugten, äußerst häufigen Einzelüberlieferungen von Abschnitten in Drucken und Handschriften bis in das 17. Jahrhundert hinein. ${ }^{16}$ Sie korrespondieren im Hinblick auf das Magnificat mit der noch ungeklärten Praxis, Wiederholungsmodelle zu komponieren (Vers 2 = Vers 8, Vers 4 = Vers 10, Vers 6 = Vers 12), ${ }^{17}$ was, wenn schon nicht auf ein Bedürfnis, so doch den Brauch schließen lässt, Einzelverse des Magnificat frei kombinieren zu können. Dem tradierten Geschichtsbild einer zyklischen Form fügt sich dies nicht ein: Hier liegt offenbar eine außerordentlich umfangreiche und anspruchsvolle Gattung vor, der die scheinbar so dominanten >großformalen Ambitionen des ausgehenden 15. und beginnenden 16. Jahrhunderts wenn nicht fremd, so doch zumindest nicht verpflichtend waren. Die formalen Ergebnisse lassen sich hier schlicht nicht von den funktionalen Konzessionen und Variablen lösen.

$16 \mathrm{Vgl}$. etwa die Sammeldrucke von Petrejus (RISM 1541 ${ }^{2}$ ), Gardano (RISM 1543 ${ }^{19}$ ) oder Montanus (RISM 154916).

17 Vgl. Winfried Kirsch, Art. »Magnificat «, in: Die Musik in Geschichte und Gegenwart, 2. Ausg., hrsg. von Ludwig Finscher, Sachteil, Bd. 5, Kassel und Stuttgart 1996, Sp. 1572-1579: Sp. 1575f. 
So scheint zusammenfassend die Untersuchung der marianischen Funktionalisierung des Magnificat, die die musikalische Interpretation erst ermöglichte, wenn nicht gar verlangte, weitaus ertragreicher zu sein als bloß seine Abschweifungen auf gattungshistorischer Ebene zu akzentuieren. Verankert in seinem Ritus, eröffnete das literarisch anspruchsvolle Magnificat Möglichkeiten zur künstlerischen Intensivierung seiner ekklesiologischen Aussage einerseits, zur Distribution der so gestifteten rituellen Kunst in sämtliche Bereiche der privaten und institutionellen Andachtskultur hinein andererseits. Dass dieses keine Sonderform von etwas Übergeordnetem, sondern eine Kunstform eigener Prägung darstellt, sollte deutlich geworden sein. Motette und Magnificat teilen kaum einen imaginären gattungsspezifischen Ort (so die Motette selbst überhaupt über einen solchen verfügt). Genau genommen sind sie nicht einmal im Sammelbegriff >mittellanger Tonstücke (»motetus «) subsumierbar, da das Magnificat seine zeitgenössischen Motetten im Umfang oft weit überragt. Am ehesten verbindet beide so etwas wie ein » motettisches Bewusstsein $"{ }_{1}^{18}$ wie Peter Gülke dies bezeichnet hat, eine Entwicklung und Interpretation von kompositorischen, oft textbezogenen Techniken und Strukturmerkmalen aus demselben zeithistorischen Fundus, der ebenso der Messkomposition offen stand. Dass die funktionalen Kontexte des Magnificat bis in die Kirchenväterzeit und die Anfänge der Liturgie überhaupt zurückreichen, weist es - anders als die Motette - als genuin liturgische und zugleich ältere Gattung aus. So teilt es eher mit der Messe des 15. Jahrhunderts einen künstlerischen Aufschwung, nämlich die Expansion musikalischer Mittel im rituellen Gefüge. Während die Messe hingegen stets eine res sacra höchsten gottesdienstlichen Ranges blieb, konnte das Magnificat seinen Ort zugleich in der privaten Frömmigkeit finden, was seinen Tendenzen zur dramaturgisch adäquaten Dehnung der psalmodischen Gestalt entgegenkam, einer Dehnung, die im Kontext einer Messe schlicht undenkbar gewesen wäre. Hier konnten nun die neuen motettischen Techniken dienstbar gemacht werden: Das »motettische Bewusstsein « polyphoner Kunst begann in rituell determinierter Musik wirksam zu werden. Wenn das Magnificat also Teil hatte an einer Phase der Normierung und Pluralisierung der Motette im 15. Jahrhundert, wie sie im vorliegenden Band diskutiert wird, so besetzt es allenfalls den Platz eines ambitionierten, ja geradezu unersättlichen Importeurs von motettischen Initiativen und Impulsen, um seinen varianten Prämissen Nahrung zu geben. Das macht es zwar keineswegs zu einer Sonderform der Motette, stellt umgekehrt aber klar, dass sich überhaupt nur im

$18 \mathrm{Vgl}$. dazu die Überlegungen zu Dufays »motettischem Bewußtsein« in: Peter Gülke, Guillaume Du Fay. Musik des 15. Jahrhunderts, Stuttgart und Kassel 2003, S. 420-435. 
»cantus mediocris « als rituell unabhängiger Form ein derart großer Fundus an Verfahren entwickeln konnte, aus dem sämtliche Gattungen des 15. Jahrhunderts schöpften. Wieviel daraus in die liturgisch fest verankerten Gattungen diffundieren konnte, hing ganz und gar von deren funktionaler Ausdifferenzierung und Verortung ab, war aber stets definiert von ihren rituellen Strukturen. Schaut man unter diesen Bedingungen auf die musikalischen Gattungen bis 1500, so zeichnet sich ab, dass ihre formale Pluralisierungsbereitschaft steigt bei Abnahme ihrer rituellen Verpflichtungen, soll heißen: Hat eine Motette keinen festen liturgischen Ort, sondern bleibt funktional variabel, so kann sie weitaus mehr Verfahren erproben und verwerfen als eine Messe oder ein Magnificat. Das klingt banal, macht aber darauf aufmerksam, dass sich derartige künstlerische Pluralisierungen nicht gegen rituelle Grenzen richten, sondern zunächst einmal nur dort entstehen, wo keine sind. Angesichts dessen wäre grundlegend über musikhistoriographische Binsenwahrheiten nachzudenken, nach denen künstlerische Entfaltung und Originalität angeblich erst aus der Herausforderung erwachse, gegen funktionale Korsette anzuarbeiten. Um 1400 begann der Ritus sukzessive, Kunst zum Zwecke des »laus Dei« einzulassen, Kunst, die bereits um ihn herum existierte und die ihn - langfristig - zu individualisieren begann. 